\title{
Pierre-Charles-Alexandre Louis' (1787-1872) Genfer Schüler und die «méthode numérique»
}

\author{
Von Eduard-Rudolf Müllener
}

Unter den vielen ausländischen Adepten von Louis sind zahlenmäßig die Nordamerikaner und Genfer am stärksten vertreten. $\mathrm{Zu}$ den amerikanischen Louis-Schülern gehören u. a. JAMES JAGKson jr. (1810-1834), der in seinem kurzen Leben eine grundlegende Arbeit über Cholera verfaßte ${ }^{1}$, WILliam W.Gerhard (1809-1872), der zusammen mit Caspar W.Pennogk (1799 bis 1894) den Typhus erforschte und 1837 das Fleckfieber davon abtrennte ${ }^{2}$, Henry I.Bodwitch (1808-1892), der Louis ins Englische übersetzte und später vor allem als Hygieniker bekannt wurde, Oliver W. Holmes (1809 bis 1894), der 1843 die Kontagiosität des Puerperalfiebers behauptete und darüber in einen Prioritätsstreit mit Semmelweis geriet ${ }^{3}$, Ashbell Smith (1806-1886), George W.Norris (1808-1875), Thomas Stewardson (1807 bis 1878) sowie Elisha Bartlett (1804-1855), welcher 1842 erneut die Abtrennung des Fleckfiebers vom Typhus unterstrich und 1844 eine zusammenfassende Darstellung der Ideen von Louis und der Pariser klinischen Schule veröffentlichte ${ }^{4}$. Nach Bartlett sind die ersten amerikanischen Forschungsergebnisse von Belang auf dem Gebiet der modernen Medizin zur Hauptsache den erwähnten Louis-Schülern sowie der Anwendung der «méthode numérique» zu verdanken ${ }^{5}$. Die Genfer Louis-Schüler waren weniger vom Schicksal begünstigt. Zwar begründeten JACOB-MARC D'Es-

1 J. JACKson jr., Cases of Cholera collected at Paris in the month of April 1832, in the wards of MM. Andral and Louis at the hospital La Pitié, Boston 1832.

2 W. W. Gerhard, On the typhus fever, Amer. J. Med. Sci. 19 (1837) 302-303.

3 O. W. Holmes, The contagiousness of puerperal fever, New England Quart. J. Med. Surg., Boston 1843, S. 503-530.

4 E.Bartlett, An Essay on the Philosophy of Medical Science, Philadelphia 1844; The History, Diagnosis and Treatment of Typhoid and Typhus Fever, with an Essay on the Diagnosis of Bilious Remittent and Yellow Fever, Philadelphia 1842.

5 Nach E.H.Ackerknecht, Elisha Bartlett and the Philosophy of the Paris Clinical School, Bull. Hist. Med. 24 (1950) 43-60, S. 49. Siehe auch W. OsLER, The influence of Louis on American medicine, in An Alabama Student and other biographical essays, 2nd impression, London/New York/Toronto 1909, S. 189-210. Über die amerikanischen Louis-Schüler siehe ferner W. AntELT, Louis' amerikanische Schüler und die Krise der Therapie, Sudhoffs Arch. 42 (1958) 291-301; W.Steiner, Some distinguished American medical students of P.-C.-A.Louis of Paris, Bull. Hist. Med. 7 (2) (1939) 783-793; 
Pine (1806-1860), Théodore-David-Eugène Maunoir (1806-1869), und Joнn Bizot (1804-1885) in Paris die «Société Médicale d'Observation», welche sich ausschließlich mit der Anwendung und Ausbreitung der Methode von Louis befaßte, mit Louis als "président perpétuel» und Chomel und ANDRAL als "présidents honoraires $;^{6} \mathrm{zu}$ den Gründungsmitgliedern gehören ferner die Genfer Antoine Baumgartner (1808-1895), Jean -JaGques Chaponnière (1805-1859) und Ami-Daniel-Félix Sestier (1803 bis 1857), so daß von den 13 Gründern 6 aus Genf stammen!? Später stieBen aus Genf noch Frédéric Rilliet (1814-1861) sowie Louis Piachaud (1824-1890), Victor Gauthier (1824-1890) und Théodore Herpin (1799 bis 1865) dazu, und auch Henri-Clermond Lombard (1803-1895) kann man zu den Louis-Schülern rechnen, wiewohl er der erwähnten Société nicht angehörte. Aus der übrigen Schweiz interessierten sich nur Jules Cossy (1818-1878) in Lavey und Hermann Lebert (1813-1878) in Zürich für die Methodik. Die Begeisterung der Genfer für Louis und seine Ideen, die sich auch darin ausdrückt, daß fast alle ihre Dissertation und andere Arbeiten Louis gewidmet haben, konnte aber in Genf nur mit Schwierigkeiten in die Praxis umgesetzt werden-denn Genf war zu klein. Allein Großstädte wie Paris mit seinen Riesenspitälern (oder ausgedehnte Epidemien, wie sie Jackson, Gerhard und Pennock sowie Bartlett zur Verfügung standen) vermochten ein derart umfangreiches Beobachtungsmaterial zu liefern, daß sich eine genaue zahlenmäßige Erfassung und Aufgliederung überhaupt lohnte $^{8}$. In dieser Situation wich d'Espine, der sich in Paris zum ersten Theore-

G. Genty, Un étudiant américain à Paris en 1833 (0. W. Holmes), Progrès médical 75 (1947) 108-109, S.108: von 138 Mitgliedern der "Société Médicale d'Observation» waren im Jahre 183316 Nordamerikaner.

6 P.-CH.-A.Louis, Avertissement, Mémoires de la Société Médicale d'Observation 1 (1837) IX: «La Société Médicale d'Observation ... a été fondée en 1832, par quelques jeunes gens, de simples étudiants en médecine, parmi lesquels je dois nommer ici MM. Marc d'Espine, Théodore Maunoir et Bizot, de Genève, comme ayant eu les premiers l'idée de cette association». (Bizot und nicht Picot, wie P.Delaunay irrtümlicherweise angibt; siehe P.Delaunay, Les doctrines médicales au début du $19^{\mathrm{e}}$ siècle. Louis et la méthode numérique, in Science, Medicine and History. Essays on the evolution of scientific thought and medical practice written in honour of Charles Singer, collected and edited by E. A. UNDERwood, 2 vol., London/New York/Toronto 1953, Band 2, S. 321-330, 324).

7 Die übrigen sieben Gründungsmitglieder sind die Franzosen Barth, BazIN, Boudin, Martins und Peyrot, der Ire EAger und der Nordamerikaner JACKson.

8 Bouillaud behauptete 1836, er habe innert 5 Jahren 25000 Fälle gesehen - eine Zahl, die fast an die damalige Gesamtbevölkerung der Stadt Genf herankommt! J.BouiLLAud, Essai sur la philosophie médicale, Paris 1836, S.318. 
tiker und Verteidiger der "méthode numérique» aufgeworfen hatte, bald einmal auf die Bevölkerungs-, Mortalitäts- und Todesursachenstatistik aus, wo ein zahlenmäßig genügend umfangreiches Material vorhanden war. Lombard beschritt einen ähnlichen Weg und fand schließlich bei der Klimatologie die nötige Vielfalt von Unterlagen für eine statistische Bearbeitung ${ }^{9}$. Immerhin produzierten die erwähnten Genfer etliche Arbeiten, in denen entweder die «méthode numérique» diskutiert wird (so vor allem d'Espine) oder eine gewisse Rolle spielt; die Herkunft aus der Louis-Schule läßt sich an den meisten ihrer Werke unschwer erkennen, und wenn sie auch nicht soviel Wertvolles hervorbrachten wie die amerikanischen Louis-Schüler, so erscheint doch eine nähere Erörterung dieser Arbeiten gerechtfertigt. Gleichzeitig soll damit eine Art «Geschichte der Genfer Louis-Schüler» vorgelegt werden, die im Gegensatz zur Geschichte der amerikanischen Louis-Schüler bis jetzt von den Historikern übergangen wurde. Dabei wird sich zeigen, daß die Genfer auch einige therapeutische Fragen mit der Zählmethode angingen und so zusammen mit vor allem französischen Kollegen (z.B. Civiale, Fuster, Malgaigne) ${ }^{10}$ verschiedene Behandlungsmethoden kritisch zu durchleuchten versuchten, ohne deswegen in therapeutische Skepsis oder gar Nihilismus zu verfallen ${ }^{11}$. Die «méthode numérique» ihres verehrten Meisters bestand letzten Endes in der konsequenten Weiterbildung der Auffassung von Pinel, Bichat und Corvisart, welche unter dem Einfluß sensualistischer (Condillac) und «ideologischer» Philosophen (CAbanis 1757-1808, Condorcet 1743-1794, Destutt de Tracy 1754-1836) die Revolution der französischen (und gesamten) Medizin hervorbrachten: den Übergang von der «konjekturalistischen» Medizin des 18. Jahrhunderts, für welche die pathologisch-anatomischen Befunde bloß «causa continua» der im Vordergrund stehenden Symptomatik waren, wobei die Krankheitsbilder je nach dem leidenden Individuum amöboid wechselnde Formen an-

9 Für biographische Angaben und die spätere Tätigkeit von d'Espine und Lombard siehe E.- R.MüLlener, Genfer Hygiene und Medizinalstatistik in der ersten Hälfte des 19. Jahrhunderts, Gesnerus 21 (1964) 154-192.

10 J. Civilale, Recherches de statistique sur l'affection calculeuse, Comptes rendus hebdomadaires des séances de l'Académie des Sciences 1 (1835) 167-177; J.J. N.Fuster, De l'application du calcul à la thérapeutique, Gazette Médicale de Paris 3 (1832) 24-27; J.-F.Malgaigne, Etudes statistiques sur les résultats des grandes opérations dans les hôpitaux de Paris, Archives générales de médecine, J.complémentaire des sciences médicales ( $3^{\mathrm{e}}$ sér.) 13 (1842) 389-418.

11 Vgl. Artelt, Anm. 5. 
nehmen konnten, in die naturwissenschaftlich orientierte Medizin des 19. Jahrhunderts, die anatomische Läsionen (oder gestörte Funktionen; Broussais) ${ }^{12}$ als «causa efficiens» der Erkrankungen betrachtete und mit gleichzeitiger scharfer Beobachtung aller klinischer Symptome imstande war, erstmals einigermaßen festumrissene Krankheitsbilder herauszuarbeiten. Dabei wurden Deduktion und Spekulation abgelehnt und einzig die von genauer Beobachtung ausgehende Induktion als Forschungsmethode anerkannt ${ }^{13}$. Nach Louis sollte nun die Induktion, das Fortschreiten von Einzelbefunden zu umfassenden Gesetzen, durch möglichst präzise Angaben zahlenmäßiger Häufigkeiten (und nicht nur durch Eindrücke) geleitet und gesichert werden. Unter dem Einfluß von Mathematikern wie LAPLACE und von Hygiene- und Sozialstatistikern wie Villermé führte Louis also das «Statistische Berichtswesen » in die Klinik ein ${ }^{14}$. Dieser mit unerschöpflicher Geduld angewandte Kunstgriff ermöglichte es ihm, die Tuberkulose als eine Gesamterkrankung des Körpers mit Beginn in der Lunge zu erkennen, den Typhus aus der amorphen Masse von Pinels «Fiebern» herauszuschälen (und gleichzeitig Broussais' Theorie von der «gastroentérite» als Ursache sämtlicher fieberhafter Erkrankungen zu erledigen) sowie den oft

12 Vgl. E. H. Ackerknecht, Broussais or a forgotten Medical Revolution, Bull. Hist. Med. 27 (1953) 320-343.

13 Vgl. dazu u. a. E.H. Ackerknecht, La médecine à Paris entre 1800 et 1850, Les Conférences du palais de la découverte, Série $\mathrm{D}, \mathrm{N}^{\circ} .58$ vom 19.4.1958; G. Rosen, The Philosophy of Ideology and the Emergence of Modern Medicine in France, Bull. Hist. Med. 20 (1946) 328-339; O.Temkin, The Philosophical Background of Magendie's Physiology, Bull. Hist. Med. 20 (1946) 10-35. Neben der rigorosen («ideologischen») Analyse gab es natürlich gleichzeitig synthetische Bestrebungen, von denen aber in den Werken jener Zeit wenig die Rede ist.

14 Statistik = zahlenmäßige Erfassung, Untersuchung und Darstellung von Massenerscheinungen. Statistisch = zahlenmäßig. Die Statistik wirkt unterteilt in das «Berichtswesen » (Häufigkeitszählungen) und die «Statistische Methodenlehre»; Louis führte nur das Berichtswesen in die klinische Medizin ein. Gezählt wurde - allerdings mehr oder weniger unsystematisch - in der Medizin seit jeher. Vgl. dazu u.a. R.H.SHrYock, The History of Quantification in Medical Sciences, Isis 52 (1961) 215-237; B. MrLt, Empirie und das statistisch fundierte biologisch-medizinische Denken in der Geschichte, Gesnerus 13 (1956) 1-28; G. Rosen, Problems in the Application of Statistical Analysis to Questions of Health: 1700-1880, Bull. Hist. Med. 29 (1955) 27-45. Gegenüber früheren «Zählern» in klinischer Medizin hatte Louis den Vorteil, ein sehr umfangreiches Krankengut beobachten und mit neuen Methoden (Perkussion, Auskulation, Gewebe- anstatt bloßer Organpathologie) untersuchen zu können. 
nur mäßigen Nutzen des seit der Antike sakrosankten Aderlasses nachzuweisen. Indem er mit seiner Methode auch therapeutische Fragen behandelte, wurde er zum Begründer der systematischen therapeutisch-klinischen Forschung, mit welcher erstmals eine experimentelle Tendenz in die praktische Medizin einzog ${ }^{15}$; man erkennt diese Tendenz unschwer z.B. an dem Umstand, daß er jeweils schon vor Beginn einer Enquète einen generellen Versuchsplan aufstellte ${ }^{16}$. Denn das Experiment (die Frage an die Natur) erfordert eben eine möglichst klare Fragestellung a priori, und wenn man Ergebnisse auszählen will, so muß schon vorher annähernd bestimmt werden, was eigentlich gezählt und in welchem Rahmen dies geschehen soll. Die sorgfältige und sehr modern anmutende Vorausplanung läßt sich bei einigen seiner Genfer Schüler recht schön nachweisen.

Allerdings überschätzte Louis seine Methode in dem Sinne, daß er ihr absolute Exaktheit zuschrieb. Davon kann natürlich keine Rede sein, wiewohl die «méthode numérique » zweifellos in jener Zeit das präziseste Instrument der klinischen Forschung darstellte. Es geriet erst nach der Mitte des 19. Jahrhunderts mit dem Aufkommen der eigentlichen experimentellen Medizin physiologischer Herkunft sowie der Mikroskopie in Vergessenheit um in unserem Jahrhundert in verbesserter Ausgabe (d.h. versehen mit den Grundlagen der statistischen Methodenlehre) wieder Auferstehung zu feiern ...

An Gegnern fehlte es Louis nicht, wobei man sie in drei Gruppen einteilen kann: die Altmodischen, die noch Konjekturalisten im Sinne des 18. Jahrhunderts waren, die Medizin als Geisteswissenschaft ansahen und sie nicht in Verbindung mit etwas so Ordinärem wie Zahlen bringen wollten; die Zeitgenossen, welche gänzlich andere Wege gingen (vor allem Broussais) sowie die Neumodischen, welche Louis' Anliegen zwar begrüßten, aber noch mehr Sicherheit wünschten und versuchten, anstelle der bloßen Häufig-

15 Vgl. dazu E.-R. Müllener, Zur methodischen therapeutisch-klinischen Forschung der «Ecole de Paris» (1800-1850), Gesnerus 23 (1966) 122-131.

16 Vgl. Louis über die Tätigkeit der «Société Médicale d'Observation»: «Quand un de ces sujets est adopté, quelqu'un présente un plan d'observation pour que les faits destinés à éclaircir le sujet proposé soient le plus complet, portent sur le plus grand nombre de points possible: puis ce plan est discuté, et comme la règle de la Société est de ne se rendre qu'à l'évidence, la discussion ne s'arrête que quand on est d'accord sur ce plan, quand on ne trouve plus rien à y ajouter ni à en retrancher.» P.-CH.-A. Louis, Avertissement, Mémoires de la Société Médicale d'Observation 1 (1837) XII. 
keitszählung die statistische Methodenlehre in die Medizin hineinzubringen $^{17}$.

Louis hat nie eine «Philosophie» seiner Methode geschrieben, sondern seine Werke sprechen lassen. Um ihre theoretischen Hintergründe und deren Darlegung kümmerten sich neben seinem amerikanischen Schüler Bartlett ${ }^{18}$ z.B. der Franzose Bruno Danvin ${ }^{19}$ sowie vor allem der Genfer d'Espine, der als der früheste Theoretiker der Zählmethode gelten kann. Daß er dabei vieles unterstreicht, was heute selbstverständlich erscheint, ist nicht erstaunlich, da er in einer Zeit schrieb, in der noch ein guter Teil der Ärzte die Medizin ausschließlich von der Philosophie her ableiten oder von vorgefaßten großen Gedankengebäuden deduzieren wollten. Die drei Initianten der «Société Médicale d'Observation», d'Espine, Maunoir und Bizot, hatten bereits im Gründungsjahr 1832 einen pikanten Triumph zu verzeichnen: Broussais wollte sich nicht davon überzeugen lassen, daß die Tuberkulose ihren Ausgangspunkt in der Lunge hat - er bestand auf der "gastroentérite». So sezierte er denn vor den Studenten einen Phthisiker mit tuberkulösen Veränderungen der Mesenterialdrüsen, ließ aber die Lunge beiseite. Die drei Genfer Freunde machten sich anschließend heimlich über dieses Organ her und konnten das «Gesetz von Louis» (Beginn der Tuberkulose in der Lunge) bestätigen ${ }^{20}$. Der Erfolg dieser Detektivarbeit veranlaßte den Theoretiker der «méthode numérique» unter den Genfer Louis-Schülern, d'Espine, zu einem vehementen Angriff auf Broussais und dessen physiologische Doktrin, welcher er vorwirft, daß sie zwar auf Broussais' Überzeugung, nicht aber auf sorgfältiger, weitreichender Beobachtung und anschlieBender zahlenmäßiger Erfassung des Beobachteten beruhe; vorsichtshalber ist diese kleine Schrift vom Studenten d'Espine nur mit «un interne des Hopitaux de Paris » unterzeichnet! Ähnlich wie Bartlett zwölf Jahre später, fährt d'Espine fort: «Les sciences les plus avancées sont celles qui contien-

17 Für die Methode waren u. a. Trousseau (der später ein Gegner wurde), Chomel, Guénau de Mussy, Capuron, Velpeau, Rochoux, Rayer; altmodische Gegner waren Dubois D'Amiens, Double, Castel, Risueno de Amador; neumodische Gegner u. a. Bouillaud und vor allem Gavarret, der denn auch 1840, gestützt auf Laplace und Porsson, die statistische Methodenlehre zumindest theoretisch in die Medizin einführte.

18 Siehe Anm. 4.

19 B.Danvin, De la méthode numérique et de ses avantages dans l'étude de la médecine, Paris 1831.

20 Nach P.Astruc, Louis (P.-Ch.-A.) (1787-1872), Les biographies médicales (Paris) 7 (1933) 245-260, 251. 
nent le plus de vérités démontrées; les sciences qui ont le plus de vérités démontrées, sont celles dont l'histoire est le moins surchargée de doctrines, et réciproquement; car la philosophie est à une extrémité de l'échelle, les mathématiques à l'autre. Quelle est la science expérimentale dont l'histoire se rapproche le plus de celle de la philosophie? Quelle est celle où les assertions contradictoires sont les plus nombreuses? J'ai presque honte de le dire! c'est la médecine. Un physicien oserait-il présenter à l'Institut une nouvelle machine, sans mettre à la disposition de ses juges tous les moyens possibles de vérification? J'ai encore honte de le dire; c'est ce qu'on fait en médecine. Quelle est la science où les expressions, je suis convaincu, il m'est démontré, c'est mon opinion, sont le plus fréquemment employées ? encore la médecine. $»^{21}$ Hier liegt also bereits «in nuce» eine «Philosophie» der Louis-Methode vor. Die kaltblütige Gleichsetzung der Medizin mit experimenteller Wissenschaft sowie die deutliche Absage an die Philosophie wird zweifellos manchen Arzt jener Tage erschreckt haben, für den die Heilkunde nach wie vor den Geisteswissenschaften nahestand.

In seiner den Lehrmeistern gewidmeten Dissertation aus dem Jahr 1833 entwickelt dann d'Espine die Prinzipien der «méthode numérique» ausführlicher, wobei er, wie weiter oben bereits angedeutet wurde, natürlich viele für uns selbstverständliche Forderungen aufstellt, die aber offensichtlich damals, zu Beginn der eigentlichen wissenschaftlichen Medizin, nicht nachdrücklich genug vorgebracht werden konnten. So schreibt d'Espine: «Il n'y a pas quinze ans encore ... un professeur aurait cru ... se compromettre en recueillant lui-même des faits; et à qui confiait-on ce travail, le plus essentiel, la pierre angulaire de l'édifice dans toute la science d'observation? à des jeunes élèves qui avaient à peine l'habitude de voir des malades, et qui, parvenus au doctorat, abandonnaient ce travail à des plus jeunes qu'eux. '22 $^{22}$ Es gibt aber keine andere Möglichkeit zur Erringung eines Fortschritts als «l'observation et l'analyse numérique des faits». ${ }^{23}$ Eine gute Beobachtung zeichnet sich dadurch aus, daß nur die wahrgenommenen Fakten und nicht die subjektive Meinung des Forschers oder bereits irgendwelche gedankliche Verarbeitungen wiedergegeben werden, und gleichzeitig muß die Schilderung so ausführlich sein, daß sie vom Leser nachkontrolliert

21 J.-M.D'Espine, De la méthode en médecine, à propos du discours que M.Broussais a lu à l'Institut, Paris 1832, S. 7.

22 J.-M.D'Espine, Essai sur cette question: comment un médecin doit-il penser? Comment doit-il agir? Paris 1833, S. 7.

${ }^{23}$ l.c., S. 9. 
werden kann. D'Espine macht sich dann lustig über Angaben wie «Nous avons trouvé à l'auscultation des signes de bronchite $»^{24}$ und verlangt mit Recht, der Forscher habe in diesem Fall die einzelnen gefundenen auskultatorischen Phänomene zu schildern und erst dann eine Diagnose zu stellen. Höhnisch fragt er: «Qui peut se contenter d'entendre dire: Le tube digestif était sain? Qu'est-ce que l'état sain du tube digestif? De combien de conditions ne faut-il pas tenir compte pour être sûr que cet état existe!» ${ }^{25}$ Zum Kapitel «Beobachtung» stellt er abschließend fest: «Une bonne description ... est la peinture d'un fait, et non pas son explication trop souvent mensongère. . ${ }^{26}$ Dann geht er zur Analyse der Tatsachen über: «La science est l'ensemble des lois qui sont contenues dans les faits; or, pour arriver à ces lois ... il reste à voir quels sont les rapports qui lient les faits, quelles sont les différences, dans quelle proportion numérique le rapport est à la différence; car une loi n'est qu'une formule qui exprime en chiffres la valeur du rapport constant qui lie entre eux un certain ordre de faits. Ce travail est l'analyse numérique.»27 Dazu braucht es eine genügende Anzahl von mit Sorgfalt registrierten Fakten derselben Art. «Puis il faut en classer les éléments sous différents chefs communs à toutes les observations afin de permettre à l'attention de s'occuper séparément et successivement du rapport qui les lie sous le point de vue d'un seul de leurs éléments: c'est dire, en d'autres termes, qu'un tableau synoptique est indispensable à ce genre de travail. Chaque colonne ayant été analysée à part, on les compare les unes aux autres; et de cette manière on a mis en rapport les faits sous chacune de leurs faces.» ${ }^{28}$ Einige Fragen erfordern zu ihrer Abklärung eine weitaus größere Anzahl von Beobachtungen als andere, wobei die nötige Anzahl der Beobachtungen mit zunehmender Kompliziertheit der Fragestellung steigt. Bei derselben Frage geben 100 registrierte Tatsachen einen viermal höheren Sicherheitsgrad als nur 25 registrierte Beobachtungen. Am schwierigsten zu beantworten ist die Frage nach der wirksamsten Therapie einer bestimmten Affektion, da sich hier sehr viele nicht konstante Faktoren wie das Alter des Kranken, der Schweregrad der Erkrankung, der Zeitpunkt des Therapiebeginns, der Allgemeinzustand des Patienten vor der Krank-

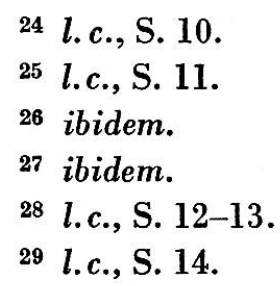


heit usw. einmischen ${ }^{30}$. Der einzig mögliche Vorwurf gegen die «méthode numérique» ist nach d'Espine der Einwand, eine hundert- oder tausendmal beobachtete Relation biete noch keine Garantie dafür, daß sich dieselbe Relation in konstanter Weise ebenfalls bei sämtlichen weiteren Beobachtungen einstellen werde sowie auch bei den nicht zur Untersuchung gelangten Fällen regelmäßig vorhanden sei. Gegen diese Attacke, die nur mittels Hinweisen auf Grundgesamtheit und Stichprobe sowie die Kriterien der Wahrscheinlichkeitsrechnung pariert werden kann, ist d'Espine schlecht gerüstet. Er meint dazu nur, er behaupte ja nicht, die «méthode numérique» sei perfekt. «Nous prétendons seulement que là où elle devient impuissante, là aussi il faut renoncer à toute espoir de découvrir la vérité.» ${ }^{31} \mathrm{Im}$ Vertrauen auf die Wirksamkeit des neuen «exakten» Forschungsinstruments macht sich d'Espine abschließend noch in jugendlichem Übermut (und zum Teil natürlich zweifellos zu Recht) lustig über «l'expérience du praticien», die das umfaßt, was jedem subjektiv am meisten Eindruck gemacht hat; über «l'expérience des siècles. On veut dire par là ce qu'on a toujours fait »; sowie über den «tact médical» als Therapeutikum, der zwar nicht abzulehnen, aber völlig unwissenschaftlich und unkontrollierbar sei ${ }^{34}$.

1834 ging dann Louis selber zur Attacke über und erledigte in seinem Examen de l'examen de M. Broussais ${ }^{35}$ dessen Ansichten über die Phthise und vor allem die "gastroentérite»; 1835 wies er nach, daß der Nutzen des Aderlasses bei entzündlichen Krankheiten nur gering ist ${ }^{36}$. Weitere theoretische Unterstützung erhält er 1836 erneut von d'Espine, der einmal mehr über die Methode schreibt und ferner in einer Besprechung von Bouillauds Essai sur la philosophie médicale (1836) die Angriffe dieses «Neumodischen» auf Louis zu parieren versucht. In seinem ersten Artikel «De la critique des faits et des opinions en médecine» umschreibt d'Espine nochmals die Kennzeichen einer guten Beobachtung: «... il faut rigoureusement observer les

30 ibidem.

${ }^{31}$ l.c., S. 15.

32 l.c., S. 17.

${ }^{33}$ l.c., S. 25.

${ }^{34}$ l.c., S. 21.

${ }_{35}$ P.-Сн.-A.Lours, Examen de l'examen de M. Broussais, relativement à la phthisie et à l'affection typhoide, Paris 1834 (J. JACKson jun. gewidmet).

${ }^{36}$ Р.-Сн.-A.Lours, Recherches sur les effets de la saignée dans quelques maladies inflammatoires etc., Paris 1835.

37 J.Boulluaud, Essai sur la philosophie médicale et sur les généralités de la clinique médicale etc. Paris 1836. 
observations pour en tirer seulement ce qu'elles renferment de vrai, c'est en quoi consiste la critique des faits ... Une première règle ... est de se placer vis-à-vis des faits qu'on va recueillir ou lire dans un état d'indifférence complète pour les résultats auxquels on doit arriver par l'analyse. Il faut avant tout vouloir ce qui est. Il découle de ce précepte que l'on ne devra pas lire avec moins d'intention de critiquer avec sévérité les faits qui sont d'accord avec ses propres opinions préjugées, que ceux qui leur sont défavorables.» ${ }^{38}$ Diese zweifellos der Forschung sehr förderliche Einstellung vermißt d'Espine bei vielen früheren Autoren und einigen Kollegen, und er bringt nun entsprechende praktische Beispiele, die er rücksichtslos zerpflückt; dabei schreckt er mit seiner Kritik auch nicht vor der Krankengeschichte eines Mitglieds der «Société Médicale d'Observation » zurück! ${ }^{39} \mathbf{E r}$ schließt das Kapitel mit dem Satz: «La critique des observations, dirigée d'après les principes dont nous venons de faire l'application, conduit nécessairement à en extraire les faits certains, et à en écarter tout ce qui est douteux.» ${ }^{40}$ Dabei kann man nach seiner Ansicht zwar jeder Krankengeschichte zumindest einige wenige sichere Anhaltspunkte entnehmen - aber die scharfe Kritik an der Beobachtung mindert doch, vor allem auf die frühere Forschung angewandt, das unbezweifelbare Wissensgut der Medizin recht bedenklich. Schließlich wendet er sich noch der Kritik der Meinungen $\mathrm{zu}$, d.h. der Art und Weise, wie nun solche eben erwähnten «gereinigten Tatsachen» zu (Lehr-)Meinungen zusammengefaßt werden sollen. Hier zählt für ihn natürlich nur die «méthode numérique»; welche Folgen ihre Nichtanwendung haben kann, macht er an einem Beispiel aus dem Werk des großen Corvisart über die Herzkrankheiten deutlich: Corvisart spricht die Meinung aus, die Berufe des Seilers und vor allem des Schneiders prädisponierten besonders zu Herzkrankheiten; zählt man aber seine ausgezeichneten Beobachtungen sorgfältig nach, so zeigt sich, daß unter 56 Fällen nur 1 Schneider und überhaupt kein Seiler vorkommt! 41

Im selben Jahre 1836 nimmt d'Espine neuerdings zu Bouillauds Essai sur la philosophie médicale Stellung. Bouillaud bringt darin einen breit angelegten medizinhistorischen Exkurs, der bei Hippokrates anfängt und

\footnotetext{
38 J.-M.D'Espine, Philosophie de la critique des faits et des opinions en médecine, J. hebdomadaire des progrès des sciences médicales 2 (1836) 325-345, 357-375, 327 (Motto der Zeitung: Le progrès est dans l'observation).

39 l.c., S. $357 / 58$.

40 l.c., S. 373.

41 l.c., S. 374-75.
} 
bei der Pariser Schule endet, zitiert häufig Laplace (sucht also mehr mathematische Sicherheit), greift aber schließlich ausgerechnet zur «méthode numérique », um die Wirkung seiner «saignée coup sur coup» mit dem nach Louis angeblich mäßigen Effekt des gewöhnlichen Aderlasses bei Entzündungen zu vergleichen! Die Gegenüberstellung ist natürlich nicht nur wegen der geringen Zuverläßigkeit der «méthode numérique» in therapeutischen Fragen unmöglich, sondern auch wegen der praktisch fehlenden Homogenität der untersuchten Patientengruppen. Immerhin «beweist» Bouillaud mit Louis' Methode die Nützlichkeit seiner eigenen «saignée coup sur coup », was ihn zu dem selbstgefälligen Ausruf bewegt: «... car enfin M. Louis n'est pas infaillible. ${ }^{42}$

Aus heutiger Sicht sind uns die Gründe für die diametral entgegengesetzten Resultate von Louis und Bouillaud hinsichtlich der Nützlichkeit des Aderlasses klar. Zu ihrer Zeit aber muß es äußerst bestürzend ausgesehen haben, daß die damals exakteste aller Methoden zu solchen Diskrepanzen führen konnte. Ebenso wie Bouillaud läßt sich auch d'Espine in der Kritik von dessen Werk zu diversen Gehässigkeiten hinreißen, und im ganzen ist sein Artikel heute nicht mehr sehr interessant. Immerhin hat auch d'Espine gemerkt, daß Bouillaud irgendwie über die «méthode numérique» hinausgelangen möchte: «... il (sc. Bouillaud) ne paraît point convaincu que l'analyse numérique soit l'unique méthode démonstrative en médecine ....\$3 Hinsichtlich des Rätsels betreffend die Nützlichkeit des Aderlasses gelangt d'Espine zu dem vernünftigen Vorschlag, die Ergebnisse Bouillauds einstweilen als Arbeitshypothese anzunehmen und sie einer möglichst rigorosen Prüfung zu unterziehen, um ihren Wahrheitsgehalt herauszufinden ${ }^{44}$.

Die praktische Anwendung der von ihm mit soviel Verve vorgetragenen und verteidigten Methode auf die Erforschung von Krankheitsbildern und Behandlungsarten gelang d'Espine allerdings dann nur bei fünf Untersuchungen, die alle ihren Ausgangspunkt noch in Paris haben. 1831 untersuchte er tierexperimentell (an Hunden) und klinisch die Relationen zwischen den Herztönen und den physiologischen Vorgängen am Herz; auf Grund sorgfältiger Beobachtungen und numerischer Analyse der Fakten schloß er, daß der erste Herzton durch die Kontraktion, der Zweite durch

42 Bouillaud, siehe Anm. 37, S. 377.

43 J.-M.D'Espine, Rezension von Bouillauds Philosophie médicale, in J. hebdomadaire des progrès des sciences médicales 4 (1836) 270-282, 330-350, 335.

44 l.c., S. 350. 
die Dilatation der Ventrikel zustande komme ${ }^{45}$. Hinsichtlich der zeitlichen Beziehung zwischen den Tönen und der Ventrikelaktion ist diese Beobachtung zweifellos grosso modo richtig - im übrigen war die verwendete Untersuchungstechnik für die subtile Fragestellung natürlich zu grob; da half auch die Anwendung der «méthode numérique» nicht darüber hinweg. 1835 legte er die Resultate einer persönlichen Befragung von 85 Pariser Frauen (kombiniert mit 200 Fällen anderer Forscher) hinsichtlich des Eintrittes der Menarche und seiner Beeinflussung durch die geographische Lage, das Klima, das Temperament (wir würden Konstitution sagen) usf. vor, wobei die Resultate recht genau mit der allgemein herrschenden Meinung über diese Fragen übereinstimmen (z.B. daß sie sich in südlichen Ländern allgemein relativ früher einstellt) ${ }^{46}$. Es beeindruckt d'Espine nicht sehr, daß die Ergebnisse seiner Enquête eigentlich wenig spektakulär ausgefallen sind. Stolz - und zweifellos mit einem gewissen Recht - erklärt er, zumindest habe er mit exakten Methoden bewiesen, was bisher bloßer Glaube gewesen sei ${ }^{47}$. Methodisch ähnliche Untersuchungen befassen sich mit dem Fluor albus und seinen Ursachen, dem Aussehen der Portio uteri bei 120 von d'Espine selber mittels des Spekulums untersuchten Frauen (Nulliparae, ältere kinderlose Frauen und Frauen mit Kindern $)^{48}$ sowie der gonorrhoischen Orchitis. Hier analysiert d'Espine zahlenmäßig seine eigenen Beobachtungen an 30 Patienten, wobei er bereits zu der heute wieder vielfach verwendeten Methode übergeht, die Schmerzintensität in Ziffern auszudrücken, um möglichst meß- und vergleichbare Resultate zu erhalten ${ }^{49}$. Auf Grund seiner Erfahrungen kommt er zu dem den damaligen Auffassungen widersprechenden Ergebnis, daß die Unterdrückung oder das spontane Erlöschen der

45 J.-M.D'Espine, Recherches expérimentales sur quelques-unes des bases qui doivent servir au diagnostic des maladies du cœur et de la circulation, Archives générales de Médecine ( $1^{\mathrm{e}}$ sér.) 27 (1831) 145-173, 172-73.

46 J.-M.D'Espine, Recherches sur quelques-unes des causes qui hâtent ou retardent la puberté, ibidem ( $2^{\mathrm{e}}$ sér.) 9 (1835) 5-18, 303-318, 13.

47 l.c., S. 18.

48 J.- M.D'Espine, Recherches analytiques sur quelques points de l'histoire de la leucorrhée, ibidem ( $2^{\mathrm{e}}$ sér.) 10 (1836) 160-183; J.-M. D'Espine, Le col de l'uterus chez la femme jeune, nullipare; influence de l'âge et des grossesses sur le col examiné au speculum, ibidem (2 $2^{\mathrm{e}}$ sér.) 10 (1836) 420-425.

49 J.-M.D'Espine, Mémoire analytique sur l'orchite blénorrhagique, Mémoires de la Société Médicale d'Observation 1 (1837) 412-495, S. 420-21: «J'ai représenté par des chiffres les expressions très peu douloureuse, peu, médiocrement, très, extrèmement, que j'ai employées pour apprécier le degré d'intensité de la douleur.» 
eitrigen Urethritis nicht der Grund für die Entstehung einer gonorrhoischen Orchitis sein können ${ }^{50}$. Damit hatte er alles noch in Paris gesammelte Material nach den Kriterien der «methode numérique» bearbeitet und wandte sich in der Folge - da ihm in Genf das für solche Untersuchungen notwendige umfangreiche Krankengut fehlte - der Bevölkerungs- und Todesursachenstatistik zu.

Henri-Clermont Lombard (1803-1895) gehörte zwar nie zur «Société Médicale d'Observation ", ist aber wohl neben d'Espine und Rilliet der bekannteste Genfer Louis-Schüler; ähnlich wie d'Espine war er nach seiner Rückkehr in die Vaterstadt (1829) infolge der engen Verhältnisse in Genf gezwungen, die reine Krankheitsbeschreibung im Stile von Louis aufzugeben, wobei er in die Klimatologie und allgemeine Hygiene auswich - in Gebiete, zu denen er schon als junger Mann eine Neigung gefaßt hatte. Seine späteren Arbeiten, vor allem die monumentale Climatologie médicale (1877 bis 1880), sind durchwegs Louis gewidmet und versuchen eine Anwendung von dessen Methode (scharf beobachten und auszählen) auf klimatologische und allgemein hygienische Probleme. Lombard hatte noch in Paris Material gesammelt zu zwei rein «numerischen» Krankheitsbeschreibungen: für seine vor allem Andral verpflichtete Dissertation Essai sur les tubercules, die er im Jahre 1827 publizierte und worin er die verschiedenen Formen der Tuberkel sehr genau beobachtet sowie auch auszählt ${ }^{51}$; ferner für seine 1830 veröffentlichten Recherches sur la pneumonie, wo auch die von d'Espine geforderten synoptischen Tafeln nicht fehlen. Lombard will hier vor allem die schon bei Morgagni auftauchende Behauptung, bei der Pneumonie sei die rechte Seite häufiger befallen, für deren Richtigkeit Chomel, Louis und Andral erste zahlenmäßig untermauerte Hinweise geliefert hatten, einer erneuten scharfen Prüfung unterziehen. Gestützt auf 968 Fälle (257 eigene, 652 bei Chomel und Andral sowie 59 bei anderen Autoren entlehnte), bei deren überwiegender Zahl eine Autopsie vorgenommen werden konnte, gelangt er zum Ergebnis von 413 rechtsseitigen, 260 linksseitigen und 195 doppelseitigen pneumonischen Befunden, wobei dieses Überwiegen der rechten Seite bei sämtlichen Altersklassen auftrat ${ }^{52}$. Er beschäftigt sich ferner mit der Altersverteilung der Lungenentzündung und findet sie relativ häu-

50 l.c., S. 494.

51 H.-C.Lombard, Essai sur les tubercules, Paris 1827.

52 H.- C. Lombard, Recherches sur la pneumonie, Bull. Soc. Méd. d'Emulation (1830) 60-72, 61 und Tabelle S. 69. 
figer bei Kindern als bei Erwachsenen ${ }^{53}$, was den damaligen Auffassungen etlicher Ärzte offenbar widerspricht ${ }^{54}$. Damit war Lombards Material erschöpft, und er ging ab 1832 zur Bevölkerungsstatistik und später zu Klimaund Seuchenhygiene über.

Die weiteren Genfer Gründungsmitglieder der «Société Médicale d'Observation ", Maunoir, Bizot, Baumgartner, Chaponnière und Sestier erlitten hinsichtlich der Anwendung der «méthode numérique» ein ähnliches Schicksal wie die beiden Hauptvertreter dieser Richtung, d'Espine und Lombard: fast jeder von ihnen brachte mit dem noch in Paris beobachteten Material eine bis zwei Arbeiten im Stile von Louis hervor - aber nach diesen vielversprechenden Anfängen und mit ihrer Rückkehr in die Vaterstadt wurde es allgemein recht still um sie, und es erfolgte in keinem Fall eine Fortsetzung der mit viel Elan begonnenen numerischen Studien. Einzelnen dieser Louis-Schüler gelang allerdings - ähnlich wie d'Espine und Lombardein Übergang auf andere medizinische Gebiete, die auch in Genf reichlich Material zur Verfügung stellten, so daß einige Alterswerke noch deutlich den Stempel der «Zählmethode» tragen; sie sind auch meist Louis gewidmet.

Théodore-David-Eugène Maunoir (1806-1869), Sohn und Neffe von bekannten Genfer Ärzten, gehörte dem «Triumvirat» an, welches den ursprünglichen Kern der «Société Médicale d'Observation» gebildet hatte (d'Espine, Maunoir und Bizot). Er studierte von 1829 bis 1833 in Paris, promovierte dort 1833 zum Doktor in Chirurgie und kehrte nach Genf zurück, wo er bis zu seinem Tode als Chirurg mit besonderer Vorliebe für Ophthalmologie wirkte; ab 1840 war er Chefchirurg am Genfer Spital ${ }^{55}$. Seine bevorzugten Pariser Lehrer waren Louis und Roux, und Louis ist auch sein einziges schriftliches Produkt von größerem Umfang, die Dissertation Essai sur quelques points de l'histoire de la cataracte gewidmet. Maunoir handelt darin seine eigenen Beobachtungen an rund 120 Starkranken im «Service chirurgical de la Charité» aus den Jahren 1830 bis 1833 nach der «méthode numérique» ab, wobei in seinem Text sogar Schreibweisen wie «73/115 der Kranken ${ }^{56}$ auftauchen, welche monstruösen Bruchzahlen er

53 l.c., S. 68.

54 l.c., S. 65.

55 A.- J.Duval, Notice sur le Docteur Théodore Maunoir, Lausanne 1869; L.Piachaud, Rapport du président sur les travaux de la Société médicale de Genève pendant l'année 1869, lu dans la séance du 5 janvier 1870, S. 1-12 (Nekrolog).

56 Th. Maunoir, Essai sur quelques points de l'histoire de la cataracte, Paris 1833, S. 78. 
dann allerdings jeweils so gut wie möglich in einfachere Verhältnisse überzuführen versucht. Maunoir gelangt zu folgenden Resultaten: als die Kataraktbildung begünstigende Faktoren kommen nur das Alter und die Vererbung in Frage, während er auf Grund seiner Untersuchung den Einfluß des Geschlechts (61 Männer und 60 Frauen von 121 Patienten), des Berufs, der Lebensführung, der Konstitution oder vorher durchgemachte Krankheiten ablehnen muß. Die langsame Herausbildung der Linsentrübung ist häufiger als die schnelle, und es brauchte in der Regel durchschnittlich 5 Jahre, bis die Patienten das Spital aufsuchten. Von 197 Extraktionsoperationen gelangen 97 (etwas weniger als 5 auf 9), die aber doch 73 der 115 operierten Kranken die Sehkraft wieder verschafften (etwas mehr als 5 Kranken auf 8), was Maunoir in dem Satze zusammenfaßt: «Les résultats de l'opération de la cataracte par extraction ont été plus favorables aux individus qu'aux yeux opérés.» Von den 82 vergeblich operierten Augen zeigten 30 einen sekundären Katarakt, 14 eine Eiterung, 19 eine vollständige Corneatrübung und 18 verschiedene Läsionen ${ }^{57}$. Man ist nicht wenig erstaunt, nach dieser leidenschaftslosen und recht modern wirkenden statistischen Auszählung (von der hier nur ein äußerst knapper Eindruck vermittelt werden konnte) einen Anhang mit «Hippocratis aphorismi» zu finden, deren Diskussion offensichtlich auch damals noch zum Pensum eines Dissertanden gehörte.

John Bızoт (1804-1885), der dritte Hauptinitiant der «Société Médicale d'Observation " studierte zur selben Zeit wie Maunoir in Paris, promovierte 1835 zum Doktor der Chirurgie, kehrte nach Genf zurück und widmete sich einer gutbesuchten Praxis; als einzige offizielle Stellung bekleidete er während sechs Jahren den Posten eines Spitalarztes ${ }^{58}$. Im Vorwort seiner Louis gewidmeten Dissertation umreißt er deren Inhalt wie folgt: «Je dois à la bienveillance de M. Louis d'avoir pu anatomiser et décrire avec soin environ cent soixante cœurs et systèmes artériels, et c'est l'analyse rigoureuse de ces cas, qui ont été comparés entre eux et comptés, dont je donne ici le sommaire. ${ }^{59}$ Gestützt auf seine Untersuchungen gibt Bizot u. a. jene Stellen der Beinarterien an, die selten von atheromatösen Veränderungen befallen sind und sich daher besonders gut zur Anbringung von Ligaturen eignen ${ }^{60}$; ferner findet er nicht traumatische arterielle Aneurysmen meist in der Knie-

57 l.c., S. 90-93.

58 Siehe den Nekrolog: Revue médicale de la Suisse Romande 5 (1885) 615-616.

59 J.Bizot, Recherches sur les altérations du système artériel chez l'homme, Paris 1835, S. 5.

60 l.c., S. 40-41. 
kehle sowie die Herzgröße von Phthisikern gegenüber andern Kranken leicht verkleinert ${ }^{61}$. Eine erweiterte Fassung seiner Dissertation veröffentlichte Bizot 1837 im Organ der «Société Médicale d'Observation», wobei er sich hier vor allem auf die Einflüsse des Alters, des Geschlechts und nicht direkt das Zirkulationssystem betreffender Krankheiten auf Herz- und Kreislauforgane konzentrierte. Wohl zu Recht bemerkt er einleitend: «Si l'on étudie les travaux des auteurs qui ont écrit sur les maladies du cœur et du système artériel, on s'étonne de n'y rencontrer nulle part une détermination précise de l'état normal; aucun pathologiste, que je sache, n'a recherché quelle influence pouvaient exercer sur l'appareil circulatoire l'âge, le sexe, les maladies, celles surtout qui affectent d'autres organes ou d'autres appareils.» Er zitiert dann Bichat und Corvisart, die beide von der Notwendigkeit durchschnittlicher Maße und Werte (besonders hinsichtlich des Herzens) schrieben, selber aber keine ausgedehnteren Untersuchungen zur Festlegung einer solchen Norm unternahmen ${ }^{62}$. Bizot wählt für seine Zwecke sorgfältig eine stets gleiche Anzahl männlicher und weiblicher Leichen aller Altersstufen von 15 bis 89 Jahren aus (diese Symmetrie kommt besonders schön zum Ausdruck in der Tabelle über Koronararterien und ihre Läsionen, S. 385), wobei 122 aus Louis' Service in der Pitié und 35 aus Kinderspitälern stammen ${ }^{63}$. Die Ergebnisse dieser enormen Fleißarbeit schildert Bizot sehr breit in drei großen Abschnitten: 1. Dimensionen des Herzens und der Gefäße im normalen Zustand, nach Alter, Geschlecht, Größe, sowie bei Krankheiten, welche nicht das Kreislaufsystem betreffen; 2. Generelle Übersicht über die krankhaften Veränderungen des Kreislaufapparates; 3. Einfluß des Alters, des Geschlechts und verschiedener Krankheiten auf die pathologischen Veränderungen des Kreislaufsystems ${ }^{64}$. Aus der verschwenderisch mit synoptischen Tafeln und zahlenmäßigen Darstellungen ausgestatteten Arbeit sei nur angeführt, daß er neuerdings die Herzen von Phthisikern gegenüber der Norm verkleinert fand ${ }^{65}$ und daß z. B. die Altersabhängigkeit der Koronarkrankheiten sowie ihr häufigeres Vorkommen bei Männern sehr schön zutage treten; es ist ferner erstaunlich, daß schon damals bei 46 Patienten unter 39 Jahren 20 mal makroskopisch sichtbare

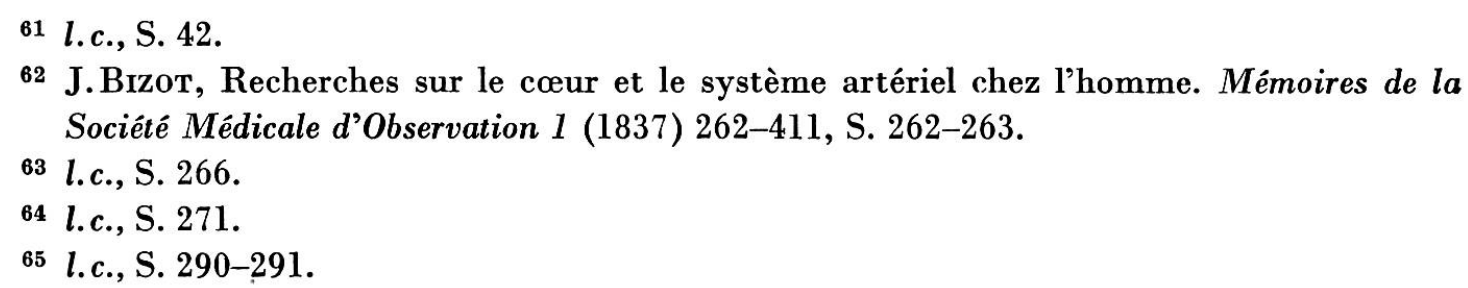


Läsionen (entweder der linken oder rechten Koronararterie, die absolute Zahl der erkrankten Herzen ist nicht eruierbar) gefunden wurden ${ }^{66}$. Bizots Arbeit ist sowohl hinsichtlich der Zielsetzung als auch der Ausführung (umfassende Beobachtung an einem nach Alter und Geschlecht sorgfältig ausgewählten Material, Auszählen und Darstellung in synoptischen Tafeln) ein Paradestück der "méthode numérique», und sie hat denn auch das uneingeschränkte Lob des Meisters gefunden: Louis erwähnt sie 1843 in der 2. Auflage seines Werkes über die Phthise und nennt Bizots Forschungen «(de) belles et profondes recherches». ${ }^{67}$ Tatsächlich kommt hier die von Louis inaugurierte genaue Vorausplanung der Untersuchungen, also die experimentelle Note, sehr schön zum Ausdruck.

Antorne Baumgartner (1808-1895), Abkömmling einer Familie aus Liestal, die sich 1722 in Genf niederließ, studierte von 1829 bis 1833 in Paris, hauptsächlich bei Dupuytren, schloß sich auch dem Kreis um Louis an, praktizierte später in Genf, befaßte sich aber hauptsächlich mit Politik und Polemik; daneben widmete er sich in vielen Zeitungsartikeln praktischhygienischen Problemen. Er hat keinerlei Arbeit im Sinne der «méthode numérique » geliefert, und seine Dissertation enthält keine einzige selbständige Beobachtung, sondern erinnert mit ihren Erörterungen sämtlicher Lehrmeinungen, aus denen der Autor zuletzt gewissermaßen eine eigene Auffassung ableitet, bedenklich an die Thesen früherer Jahrhunderte ${ }^{68}$.

Dasselbe gilt für die Dissertation von Jean -Jacques Chaponnière (1805 bis 1859) ${ }^{69}$, das einzige «praktisch »-medizinische Werk dieses Genfer LouisSchülers, der 1826 bis 1832 in Paris studierte und anschließend in Genf praktizierte; er beschäftigte sich daneben hauptsächlich mit Medizingeschichte $^{70} .1853$ nahm er sein Dissertationsthema nochmals unter diesem Blickwinkel auf ${ }^{71}$ - was 1832 noch (mit Aphorismen von Hippocrates ge-

66 l.c., S. 386-387.

67 P.- CH.-A.Lours, Recherches anatomiques, pathologiques et thérapeutiques sur la phthisie, $2^{\mathrm{e}}$ éd., Paris 1843, S. 60, und Bulletin de l'Académie Royale de Médecine 1 (1836/37) 741 (Louis in der Diskussion der Akademie über medizinische Statistik).

68 Siehe dazu A.-J.Baumgartner, Le Docteur Baumgartner. Souvenirs de sa vie et de ses écrits, Genève 1895, sowie E.- R.Müllener, Anm. 9; A. Baumgartner, Dissertation sur le siège de l'hystérie, Paris 1833.

69 J.-J. Chaponnière, Essai sur le siège et les causes des névralgies de la face, Paris 1832.

70 z.B. J.-J. Chaponnière, Les léproseries de Genève au $15^{\mathrm{e}}$ siècle, Genève 1841; J.-J. CHAPONNIÈRE und L.SORDET, Les Hôpitaux de Genève avant la réformation, Genève 1844.

71 J.-J. Chaponniène, Recherches historiques sur les névralgies de la face, Recueil des travaux de la Société Médicale de Genève 1 (1853) 67-118. 
schmückte) Aktualität gewesen war, trat zwanzig Jahre später eindeutig in die Sphäre des Historischen ein. Und doch ist diese kurze sachliche Übersicht über Schilderungen der Gesichtsneuralgie von Hippocrates bis zur Neuzeit viel nützlicher als die früheren Versuche, aus vielen Lehrmeinungen eine allgemein gültige Lehre herauszulesen. Denn nun betreibt auch Chaponnière eine Art Beobachtungsmedizin - indem er seine Aufmerksamkeit den Beobachtungen vergangener Jahrhunderte zuwendet und deren brauchbare Anteile heraushebt, unbelastet von der jeweils dahinterstehenden oder darum herum aufgebauten zeitbedingten Theorie. Er wendet also hier wenigstens andeutungsweise das Verfahren an, von dem d'Espine 1836 geschrieben hatte: «observation de l'observation», d.h. die sorgfältige Sichtung früheren Erfahrungsgutes auf seine medizinische Brauchbarkeit im Lichte der jetzigen wissenschaftlichen Grundsätze. In gewissem Sinne ist also auch Chaponnière der «médecine d'observation » verpflichtet und damit Empiriker; er fügt den übrigen Forschungsmethoden der Autopsie und des (zahlenmäßig erfaßten) Ähnlichkeitsverfahrens die Methode der geschichtlichen Betrachtung bei ${ }^{72}$.

Das sechste Gründungsmitglied der «Société Médicale d'Observation», Ami-Daniel-Félix Sestier (1803-1857; gelegentlich auch «Sestié»geschrieben) promovierte 1832 in Paris und blieb zeitlebens dort, aufsteigend vom Interne über Chef de Clinique (am Hôtel Dieu bei Chomel) zum Professeur agrégé. Seine frühen Arbeiten, meist Kompilationen anderer Autoren. sind nicht sehr originell ${ }^{73}$; einzelne pathologisch-anatomische Schilderungen zeigen aber, daß Sestier auch ein guter Beobachter und echter Louis-Schüler war ${ }^{74}$. Erst 1840 fing er dann im Stile des Lehrmeisters zu

72 Siehe dazu B.MILT, Empirie und das statistisch fundierte biologisch-medizinische Denken in der Geschichte, Gesnerus 13 (1956) 1-28, S. 5/6: «Da die Erfahrungsmöglichkeit eines einzelnen Arztes aber eine begrenzte ist, seltenere Fälle ihm nicht in genügender Anzahl zu Gesicht kommen ... benützt er auch die Erfahrungen anderer Ärzte, wie sie in der Literatur niedergelegt sind. So tritt als Forschungsmittel neben die Autopsie die Geschichte ... In diesem ,Dreifu $\beta^{6}$ der Autopsie, der Geschichte und des Ähnlichkeitsverfahrens als Hypothesenlieferant sahen die Empiriker ihre Forschungsmethode ...»

73 z.B. F. Sestier, Propositions de médecine et d'anatomie pathologique, Paris 1832 (Diss.; besteht aus einzelnen Lehrsätzen); F. Sestier, Des dyspnées intermittentes, Paris 1832 (Kompilation); F. Sestier, Jusqu'à quel point la percussion et l'auscultation ont-elles éclairé le diagnostique des maladies aiguës et chroniques du cœur? Paris 1835 (Kompilation); F. Sestier, Des causes spécifiques des maladies, Paris 1838 (Kompilation).

${ }^{74}$ Etliche Diskussionen einzelner Fälle und pathologisch anatomischer Befunde sind geschildert z. B. in Bulletins de la Société Anatomique de Paris 4 (1829) 33; 7 (1832) 25, 28, 42,$53 ; 8$ (1833) 32, 63-64, 106, 130, 197, 217, 222. 
publizieren an; sein Traité sur la pneumonie, in welchem er eigene Beobachtungen mit solchen zuverläßiger Kollegen (u.a. von Rilliet, einem weiteren Genfer Louis-Schüler, von dem noch die Rede sein wird) vereinigt, erinnert in seinen endlosen minutiösen Detailschilderungen durchaus an Louis, und auch zu Tabellen geordnete zahlenmäßige Angaben fehlen nicht ${ }^{75}$. Dabei entschuldigt sich der Autor im Vorwort noch dafür, daß er nicht zu viele Details habe bringen können, um nicht ins Uferlose zu geraten! ${ }^{76}$ Ein ähnliches Verfahren (Kombination von eigenen Beobachtungen und solcher zuverläßiger Kollegen) wendet Sestier auch an zur Bewertung des Nutzens der Bronchotomie bei ödematösen Veränderungen des Larynx ${ }^{77}$; in Fällen von leichter Entzündung des Larynx bei ansonst einigermaßen gesunden Individuen findet er die Operation in vier von fünf Malen erfolgreich, in Fällen mit schweren Läsionen des Larynx bei bereits seit längerer Zeit kranken Individuen nur in einem auf neunzehn Male. Insgesamt verstarben von 102 operierten Männern 83, von 44 operierten Frauen $28^{78}$ - hier sprechen die Häufigkeitsziffern wirklich eine deutlichere Sprache als «viel, wenig, wie üblich» und lassen uns auch noch heute ganz genau die relative Ohnmacht der damaligen Ärzte vor solchen Prozessen erkennen. 1852 gab Sestier eine stark erweiterte Fassung dieses Artikels in Form eines Buches heraus, welches nun Louis gewidmet ist ${ }^{79}$; er stützt sich auf 132 eigene klinisch und autoptisch beobachtete Fälle ${ }^{80}$ sowie Berichte anderer Autoren (u. a. von Louis Piachaud, einem weiteren Genfer Louis-Schüler) ${ }^{81}$, so daß ein Total von 274 Patienten zusammenkommt. Eine synoptische Übersicht über 149 zeigt interessanterweise, daß von 89 Larynxentzündungen bei Erwachsenen nur 3 offensichtlich durch Diphtherie bedingt waren, dagegen an

75 Leçons de Clinique Médicale faites à l'Hôtel-Dieu de Paris, par M. le Professeur A.F. Chomel, recueillies et publiées par F.Sestien, tome $3^{\mathrm{e}}$ (Pneumonie), Paris 1840, S.448 bis 450 .

76 l.c., S. VI.

77 F.Sestier, De la bronchotomie dans les cas d'angine laryngée œedémateuse, Archives générales de Médecine (4 ${ }^{\mathrm{e}}$ sér.) 23 (1850) 385-419 und 24 (1850) 35-64, 297-313, 441-466, 385: die selbstbeobachteten Fälle umfassen 168, wovon 36 operiert wurden - laut Sestier die größte bisher von einem Autor überblickte Serie.

78 l.c., S. 402.

79 F.Sestien, Traité de l'angine laryngée œéémateuse, Paris 1852, S. XII: «M. le docteur Louis, dont le jugement sévère et l'habileté clinique sont si hautement reconnus, a eu l'extrème bonté de lire notre manuscrit et de nous communiquer ses réflexions.»

80 l.c., S. VII.

81 l.c., S. XI. 
die 50 durch Tuberkulose und Syphilis ${ }^{82}$. Seinen Höhepunkt fand Sestiers medizinalstatistisches Schaffen im Sinne der «méthode numérique», als er (wiewohl in Paris tätig) das Gebiet der Krankheitsbeschreibung verließ und sich einem noch reichhaltigeren Material zuwandte, nämlich dem Phänomen des Blitzes und seinen sämtlichen Auswirkungen. Sestiers diesbezügliche Beobachtungen und aus der ganzen Welt kompilierten Schilderungen wurden nach seinem Tode unter dem Patronat der beiden Hauptvertreter der Medizinalstatistik um die Mitte des letzten Jahrhunderts, Louis und Gavarret, veröffentlicht. Das monumentale Werk ${ }^{83}$ stellt sich würdig neben Lombards Climatologie médicale und D'Espines Essai de statistique mortuaire comparée.

Neben d'Espine und Lombard ist von den Genfer Louis-Schülern zweifellos Frédéric Rilliet (1814-1861) der bedeutendste; er sowie Piachaud, Gautier und Herpin stießen nach 1832 zur «Société Médicale d'Observation». Rilliet studierte von 1833 bis 1840 in Paris als Schüler von Louis, Biett und Guersent, verbrachte nach Studienabschluß drei weitere Jahre am «Hôpital des enfants malades» und kehrte dann als praktischer Arzt mit speziellem Interesse an Pädiatrie nach Genf zurück. Er publizierte an die fünfzig Arbeiten über meist pädiatrische Themen und Tuberkulose ${ }^{84}$. Seine Dissertation über den damals noch wenig erforschten Typhus bei Kindern scheint auf den ersten Blick kaum einen Einfluß der "méthode numérique» aufzuweisen; dies rührt aber daher, daß sie nur einen Auszug aus dem für den «Concours de la médaille d'or des hôpitaux» eingereichten Manuskript Rilliets darstellt, aus welchem er zwecks Kürzung «toutes les observations et les tableaux analytiques», also den ganzen Apparat der «Zählmethode», wegstrich $^{85}$. Sein nur drei Jahre später erschienenes Hauptwerk über die Kinderheilkunde (zusammen mit seinem Pariser Kollegen und Freund Barthez) bringt seine Verbundenheit mit Louis und dessen Methode deutlicher zum Ausdruck. Im Vorwort schreiben die Autoren: «Nous avions terminé la partie la plus difficile de notre travail en achevant de recueillir nos

82 l.c., S. 142.

83 F.Sestier, De la foudre. De ses formes et de ses effets sur l'homme, les animaux, les végétaux et les corps bruts. Des moyens de s'en préserver et des paratonnerres, 2 Bände, Paris 1866. Es galt noch in unserem Jahrhundert als Standartwerk und wird zitiert u.a.z.B. bei F.PANse, Über die Schädigung des Nervensystems durch Blitzschlag, Monatsschrift für Psychiatrie und Neurologie 59 (1925) 323-354, 354.

84 Siehe A.-J. Duval, Le docteur Rilliet. Sa vie et ses œuvres, Genève 1861.

85 F. Rilliet, De la fièvre typhoide chez les enfants, Paris 1840, S. 5. 
observations; restait encore la tâche de les analyser. Opération longue et fatigante, l'analyse des faits a l'avantage de faire saisir les rapports qui les unissent et les différences qui les séparent. M. Louis auquel la science est redevable de l'application rigoureuse de cette méthode à la médecine, a insisté sur les règles à suivre pour les mettre en pratique. Nous avons, à son exemple, réuni les cas de la même espèce dans de grands tableaux analytiques; puis, lorsque nous avons voulu étudier une maladie en particulier, nous avons dressé pour chaque partie de l'observation des tableaux secondaires dans lesquels les phénomènes pathologiques étaient envisagés sous toutes leurs faces. Pour les maladies importantes, nous avons fait ce travail chacun à part; lorsqu'il a été terminé, nous avons comparé et contrôlé les résultats auxquels nous étions arrivés; nous avons ensuite partagé également les divers sujets pour les soumettre à une rédaction définitive.

Désireux d'imprimer à notre travail le cachet de la vérité, nous avons dû appuyer nos assertions sur deux ordres de preuves, l'analyse numérique et les observations particulières. Afin de donner la mesure de notre expérience personnelle, nous avons eu d'indiquer pour chaque monographie le nombre des cas qui ont servi à sa composition. ${ }^{86}$

Dabei konnten die Autoren nicht alle numerischen Resultate in extenso bringen, sondern mußten aus Platzmangel viele davon durch kurze Auszüge ersetzen, worauf sie ausdrücklich hinweisen. Aber auch Rilliet teilte das Schicksal seiner nach Genf zurückgekehrten Kollegen: mangels Patientenmaterial sind seine Schriften nach 1843 meist Beschreibungen von einzelnen oder von einigen Fällen, bei denen eine Anwendung der Zählmethode nicht in Frage kam; auch die weiterhin andauernde Freundschaft und enge Zusammenarbeit mit dem in Paris gebliebenen Barthez half ihm nicht über diese Klippe hinweg, da die numerische Analyse nur bei der Applikation auf eigene Beobachtungen wirklich Früchte tragen konnte. Sehr gut lassen sich diese Umstände am Beispiel der «méningite franche» (nicht-tuberkulöse Meningitis) darlegen: in der ersten Auflage von 1843 verfügten die Autoren über 5 eigene und 1 fremde Beobachtung dieser (gegenüber der damals häufig vorkommenden tuberkulösen Meningitis) eher seltenen Affektion $^{87}$. Zehn Jahre später, in der zweiten Auflage (1853) sind erst 6 neue ei-

${ }^{86}$ F. Rilliet und E. Barthez, Traité clinique et pratique des maladies des enfants, 2 Bände, Paris 1843. Zitiert nach der 2. Auflage 1853, Band 1, S. XIII (Abdruck des Vorworts zur 1. Auflage).

87 l.c., 1843, Band 1, S. 627. 
gene Beobachtungen dazugekommen ${ }^{88}$; dabei ist anzunehmen, daß sich weder Rilliet noch Barthez (der sich allerdings weniger auf Hirnhautentzündungen spezialisiert hatte als Rilliet, einen solchen Fall in ihrer Umgebung haben entgehen lassen. Zweifellos wäre es Rilliet mit seinem speziellen Interesse für «méningite franche» in Paris binnen zehn Jahren gelungen, eine weit größere Anzahl von Fällen zu beobachten.

Von Rilliets Kollegen Louis Piachaud (1824-1890), ebenfalls Mitglied der "Société Médicale d'Observation », ist in unserem Zusammenhang nicht viel zu berichten, da er nichts mit der «méthode numérique» in Verbindung stehendes veröffentlicht hat ${ }^{89}$; er studierte von 1844 bis 1852 in Paris, wo er sich als Schüler von Velpeau und Nélaton hauptsächlich chirurgisch ausbildete, aber auch Charcot schätzte. Er kehrte 1852 nach Genf zurück, betätigte sich hauptsächlich praktisch und war von 1866 bis 1870 Chefchirurg des Kantonsspitals.

Horace - Charles -Victor Gautier (1824-1890), der Vater des Medizinhistorikers Léon Gautier, studierte von 1841-1843 in Zürich, dann bis 1850 in Paris unter Louis, Blandin und Guéneau de Mussy; zu seinen Freunden zählten Charles Robin, Verneuil und Broca. 1850 kehrte er nach Genf zurück und war vor allem chirurgisch und obstetrisch-gynäkologisch tätig ${ }^{90}$. Er verfaßte eine große Anzahl kurzer Publikationen über Probleme aus diesen Fachgebieten. An seinen präzisen Beobachtungen und Schilderungen erkennt man unschwer den Louis-Schüler, wenn es ihm auch nicht möglich war, die "méthode numérique» in einigem Ausmaß zur Anwendung zu bringen. In seinem Nachlaß fanden sich interessanterweise gedruckte und von Gautier beschriebene Originalkrankenblätter aus dem Jahre 1849, herausgegeben von der «Administration générale de l'assistance publique à Paris». Handschriftlich ist eingetragen: «Hôpital de Lourcine, Service de Guéneau de Mussy». Die obere halbe Seite bietet Platz für die «Histoire de la maladie» (zum Teil ausgefüllt mit anamnestischen Angaben), die untere

${ }^{88}$ l.c., 1853, Band 1, S. 94. Bezüglich Rilliets Meningitisforschung siehe E.-R.MüLLENER, Six Geneva Physicians on Meningitis, Journal of the History of Medicine and Allied Sciences 20 (1965) 1-26, S.21 und 25.

89 In seiner Dissertation Des déviations de l'utérus à l'état de vacuitê (Paris 1852), die 13 eigene Fälle enthält, bemerkt er einleitend: «J'ai eu recours à l'observation, parce que je pense que c'est la seule voie qui puisse conduire à des résultats positifs ... mais je dois dire que le nombre des malades qui se sont présentées à mon observation n'est peut-être pas aussi considérable que je l'aurais désiré» (l.c., S. 5-6).

${ }^{90}$ Siehe C.Picot, Le docteur Victor Gautier. Notice biographique, Revue médicale de la Suisse romande 10 (1890) 122-130. 
halbe Seite für den Verlauf («Date, Observations, Traitement externe, Traitement interne, Hygiène»). Das vorgedruckte Blatt für den aktuellen Status umfaßt 4 Seiten; die gedruckten Punkte der Untersuchung lassen an Genauigkeit und Vollständigkeit nichts zu wünschen übrig und das Schema könnte heute noch ohne weiteres verwendet werden. Dasselbe gilt vom vorgedruckten Bogen für die Sektionsbefunde, welcher 8 Seiten umfaßt. Die Erziehung zu genauer Beobachtung, wie sie in Paris um 1850 gepflegt wurde, kann also durchaus vor den heutigen Versuchen in dieser Richtung bestehen. Gautiers Dissertation von 1850 ist Louis gewidmet, und stolz bekennt er sich als Mitglied der «Société Médicale d'Observation». Die These enthält aber nur neun vom Autor selber beobachtete Fälle, was die Anwendung der «méthode numérique» überflüssig machte; Einzelheiten zur pathologischen Anatomie der Gefäßnaevi erhielt der Autor von einem andern Mitglied der «Société», Hermann Lebert, zur Verfügung gestellt ${ }^{91}$. In zwei größeren Arbeiten aus der langen Reihe von Gautiers Publikationen finden sich da und dort Andeutungen einer «numerischen» Behandlung des Stoffs; so verfaßte er 1869 eine Monographie über den Retropharyngealabszess, in welcher er sehr sorgfältig die primären Tatsachen aufsucht und immer wieder auszählt; auf Grund dieser Zählungen kommt er zu einigen interessanten Schlüssen, wie z.B.: «Je suis arrivé, par des relevés statistiques, à démontrer la fréquence relativement très grande de ces abcès dans la première année de la vie. ${ }^{92}$ In einer Monographie von 1884 versucht er an Hand von Quételets Normen für den menschlichen Körper die von ihm (und andern Forschern) bei frühreifen Mädchen beobachteten Abweichungen genauer (d.h. zahlenmäßig) zu fassen ${ }^{93}$. Interessant ist ferner, daß er 1867, beeindruckt von Semmelweis' Erfahrungen in der Geburtshilfe, auf Grund einiger eigener Beobachtungen (allerdings ohne jegliche diesbezügliche Statistik) und von gelegentlichen Bemerkungen anderer praktischer Chirurgen die von Semmelweis in der Geburtshilfe ausgearbeitete These von der Ansteckung des Patienten durch den Operateur in die Chirurgie übernahm: «... l'infection occasionnée par le contact des mains ou des vêtements des chirurgiens et de leurs aides, est probablement la cause principale des acci-

91 V. GAUTIER, Considérations et observations relatives à l'anatomie pathologique, à la marche et au traitement des tumeurs érectiles cutanés, Paris 1850, S. 9.

92 V. GAUTIER, Des abcès rétro-pharyngiens idiopathiques ou de l'angine phlegmoneuse, Genève et Bâle 1869, S. VI.

93 V.GAUtier, De la menstruation et de la maturité précoces des hémorrhagies génitales des petites filles, Genève 1884. 
dents consécutifs aux opérations chirurgicales ... Grâce aux nombreuses statistiques qui se font maintenant dans presque tous les hôpitaux d'Europe, les termes de comparaison seront faciles à établir, et ce qui n'est jusqu'à présent qu'une affirmation, sera devenu une vérité parfaitement démontrée.» Er empfiehlt folgende generelle Maßnahmen: «1. Ne pas permettre l'exercice de la chirurgie active aux personnes qui sont habituellement en contact avec les cadavres ...2. Introduire dans les services de chirurgie des réglements sévères pour prévenir l'infection des opérés et des blessés. » ${ }^{94}$ Unter Punkt 2 versteht er vor allem die strikte Reinhaltung des Operationssaales und der Hände des Operateurs mittels desinfizierender Waschungen (Chlorwasser und Seife). Dieser frühe Vorschlag eines Provinzarztes, eine Art chirurgischer Asepsis einzuführen, ging allerdings unter in der im selben Jahre 1867 von Lister propagierten Antisepsis; dabei ging Lister, der Semmelweis' Lehre nicht kannte, von der Luftkeimtheorie Pasteurs aus, übertrug sie auf die Wundkrankheiten und kam so zur antiseptischen Karbolsäurepraxis. Hätte der Vorschlag des Genfer Chirurgen und vor allem die von ihm vorgeschlagene statistische Überprüfung mehr Gehör gefunden, so wäre möglicherweise der Chirurgie der Umweg über die Antisepsis erspart geblieben!

Théodore -Joseph - Dieudonné Herpin (1799-1865) aus Carouge bei Genf studierte von 1816 bis 1823 als Schüler von Lænnec in Paris; dann kehrte er nach Genf zurück, gründete 1823 die heute noch bestehende «Société Médicale de Genève " ${ }^{95}$ und widmete sich einer ausgedehnten Praxis, wobei ihn besonders die Epilepsie und andere «nervöse » Störungen interessierten. 1856 verlegte er seinen Wohnsitz nach Paris, wo es mehr nervöse Leiden zu behandeln gab; er starb 1865 in Paris ${ }^{96}$. Der «Société Médicale d'Observation », also dem Kreis um Louis, trat er erst 1852 bei. Allerdings muß er schon früher die «méthode numérique» gekannt haben und von ihr fasziniert worden sein, denn das 1850 der Académie des Sciences in Paris eingereichte Hauptwerk Du pronostic et du traitement curatif de l'épi-

94 V.GaUtier, De la cause principale et de la prophylaxie des accidents consécutifs aux opérations chirurgicales, Bulletin de la Société Vaudoise de Médecine 1 (1867) 213-223, S. 221-222.

95 Siehe J. Olivier, Les sociétés genevoises de médecine, Revue médicale de la Suisse Romande 57 (1937) 664-686. In den Dezennien nach 1800 hatte Genf 6 verschiedene medizinische Gesellschaften aufzuweisen, von denen aber nur die 1823 gegründete «Société Médicale de Genève» bis heute überlebte.

96 E. Dufresne (Nekrolog von T. Herpin), Compte-rendu des travaux de la Société Médicale de Genève pour 1865, S. 28-33. 
lepsie (publiziert Paris 1852) ist ganz im Geiste von Louis geschrieben: unter dem Motto «exactitude et persévérance» schildert Herpin 68 selbst beobachtete Fälle, die er während eines Zeitabschnittes von rund 15 Jahren so genau wie möglich verfolgte. Die gesammelten Fakten unterzieht er dann der numerischen Analyse und versucht, die Einflüsse des Alters, des Geschlechts, der Konstitution, konkomitierender oder vorangegangener Krankheiten, der Heredität, der Menstruation, der Schwangerschaft, der Intelligenz der Patienten, ihrer sozialen Position usf. näher zu bestimmen. Am meisten interessiert war er an der Therapie; dies geht auch aus seinen recht zahlreichen kleineren Publikationen hervor, die meistens der Behandlung verschiedener Leiden gewidmet sind. Sehr schön kommt dabei die A-prioriFestsetzung des Versuchsplans zum Ausdruck. So schreibt Herpin z. B.: «... nous avons presque toujours mis un grand soin à nous abstenir, dans nos traitements, de toute complication qui pût jeter du doute sur la cause du résultat. Ainsi ... nous n'avons jamais donné deux remèdes à la fois. „ ${ }^{97}$ Die Ergebnisse der Therapieversuche sehen folgendermaßen aus: am besten wirkte ein Pulver aus der Wurzel von Selinum palustre Linné (5 von 6 Fällen mit guter oder mäßiger Prognose geheilt oder 86 auf 100 in der Schreibweise von Herpin); es folgt das Zinkoxyd, welches unter denselben Umständen 28 von 36 oder 78 von 100 Patienten heilte. Daran schließt sich das ammoniakalische Kupfersulfat an, das 4 von 7 oder 57 von 100 Epileptikern Erleichterung verschaffte. Zuletzt kommt der Baldrian, der in 5 von 9 oder 55 von 100 Fällen wirkte. Sämtliche andern versuchten Medikamente trugen wenig oder nichts zur Heilung bei; am empfehlenswertesten sind von ihnen nach den Erfahrungen von Herpin noch Silbernitrat, Pulver von einem gerösteten Maulwurf(!), Extractum Belladonnae sowie Asa foetida. Er ist sich darüber klar, daß seine Aufstellung von Therapieerfolgen nur bedingten Wert hat: «Mais il ne faut point oublier que, pour trois de ces médicaments, la conclusion repose que sur un nombre de faits qui, pour chacun, n'est que le tiers ou le quart des cas où a été administré le zinc. L'ordre que nous venons d'établir ne doit être, en conséquence, considéré que comme provisoire. . ${ }^{98}$ Die von Herpin bei Fällen mit guter oder doch zumindest mäßig guter Prognose mittels Zinkoxyd erzielten Erfolge konnten denn auch an hospitalisierten Patienten nicht reproduziert werden ${ }^{99}$. $\mathrm{Zu}$ diesem

97 Tн. Herpin, Du pronostic et du traitement curatif de l'épilepsie, Paris 1852, S. 607.

98 l.c., S. 613.

99 Zitiert nach Dufresne (siehe Anm. 96), S. 32; siehe ferner Th. Herpin, Pronostic et traitement de l'épilepsie, L'union médicale 7 (1853) 230-31, 233-34, 230. 
Umstand bemerkt Herpin ein Jahr später mit Recht, die Spitäler behandelten hauptsächlich inveterierte Fälle, während er in der Privatpraxis mehr frische Fälle mit besserer Prognose sehe ${ }^{100}$. Er ist sich auch der Tatsache bewußt, da $\beta$ viele seiner numerischen Ergebnisse auf schwachen Fundamenten ruhen ${ }^{101}$; schuld daran ist - wie er ausdrücklich betont - die zu kleine Anzahl vergleichbarer Fälle und nicht etwa ein Versagen der Methodik. Im Vorwort des Werkes von 1852 legt er ausführlich und mit Beispielen nicht geizend, seinen diesbezüglichen Standpunkt dar, wobei sich neuerdings eine kleine Theorie der «méthode numérique» ergibt; zählen ist für ihn eine Selbstverständlichkeit: «Au fond, tout le monde compte et doit compter. Pour choisir entre deux traitements, il faut comparer les résultats : comment le faire sans compter? ${ }^{102}$ Das einzige, was man der sorgfältig durchgeführten Zählmethodik vorwerfen kann, ist «sa difficulté et la longueur». So ist trotz aller (ihm selber durchaus bewußter) Mängel Herpins Arbeit ein erster Versuch, die Epilepsietherapie mit gewissermaßen naturwissenschaftlichen Methoden (Vorausplanung, experimentell-klinische Tendenz) zu überprüfen. Nicht zuletzt auf Grund der angewandten strengen Methodik verlieh ihm die Pariser Académie des Sciences am 16.12.1860 einen Preis von 1500 Francs ${ }^{104}$; umgekehrt schreibt sein Nekrograph bereits 1865 von «la méthode numérique dont il (sc. Herpin) abuse ...». ${ }^{105}$ Daraus läß sich ersehen, wie rasch die numerische Methode in der von Louis angewandten und propagierten strengen Form in Mißkredit geriet. Anschließend an die Vorarbeiten von Pinel, Corvisart und Lænnec hatte sie ihre Blütezeit und Erfolge gehabt und wurde nun in der Mitte des 19. Jahrhunderts von neuen Erscheinungen auf dem Felde der Medizin abgelöst: dem Mikroskop (Histo-

100 ibidem, S. 231.

101 Th. Herpin, l.c., siehe Anm. 97; auf S. 331-332 schließt er aus einer Serie von 31 männlichen und 37 weiblichen Epileptikern, daß die Krankheit bei Frauen häufiger sei. (Heute ist man eher der umgekehrten Ansicht, vgl. M. Reicharot, Allgemeine und spezielle Psychiatrie, 4. Auflage, Basel 1955, S. 447.)

102 l.c. (siehe Anm. 97), S.17.

103 l.c., S. 21.

104 «L'importance du sujet étudié par M. Herpin, la sévérité de la méthode qu'il a suivi pour observer et apprécier les faits, et enfin l'intérêt de plusieurs des résultats auxquels il est arrivé, ont paru à la commission mériter à ce médecin une récompense de quince cents francs. » (Académie des Sciences, Rapport sur les prix de médecine et de chirurgie pour les années 1849 et 1850; Séance publique du lundi 16 décembre 1850; dieser Rapport ist Herpins Buch vorangeheftet.)

105 l.c. (siehe Anm. 96), S. 32. 
pathologie und Bakteriologie) sowie der experimentellen Grundlagenforschung vor allem physiologischer Provenienz.

Herpin arbeitete unermüdlich an dem Problem der Epilepsie weiter, wobei ihn neben der Therapie ${ }^{106}$ die Zerlegung des Epilepsie-Komplexes in verschiedene Untergruppen (mit verschiedener Prognose und Behandlungsmöglichkeit) ${ }^{107}$ sowie vor allem die Früherfassung der Fälle interessierten. In der Frage der Behandlungs- und Beeinflussungsmöglichkeit des Leidens (welches damals nach den Spitalerfahrungen als praktisch unheilbar galt) blieb er zeitlebens optimistisch ${ }^{108}$; merkwürdigerweise erwähnt er mit keinem Wort je das von Sir Charles Locock (1799-1875) in London von 1851 bis 1857 in die Epilepsiebehandlung eingeführte Bromkalium, welches als erstes Spezifikum gegen diese Krankheit gelten darf. Herpins zweites Hauptanliegen, die Aufspaltung des Epilepsiekomplexes in verschiedene Unterformen zum Zwecke einer differenzierten Behandlung, Prognosenstellung und möglichst frühen Erfassung der Kranken fand ihren Niederschlag in seinem 1867 posthum veröffentlichten Werk Des accès incomplets d'épilepsie, in dem er sich als ausgezeichneter Beobachter erweist und seine eigenen Erfahrungen an 300 Patienten beschreibt, bei denen nicht die Anfälle, sondern die Äquivalente im Vordergrund stehen; wir verdanken ihm dabei die erste klare Beschreibung der psychomotorischen oder Schläfenlappenepilepsie, als deren Prodrome Herpin u.a. «des perturbations de l'intelligence, avec ou sans hallucinations» schildert ${ }^{109}$.

106 Th. Herpin, Pronostic et traitement de l'épilepsie, L'Union médicale 11 (1857) 408-9, 413, 453, 492, 525, 573, 620-621, S. 408: «Il y a en France cent mille épileptiques dont la plupart sont aujourd'hui incurables parce qu'on n'a pas cru a leur curabilité... Pour réussir dans l'épilepsie, il faut avant tout attaquer le mal à une époque aussi rapprochée que possible de son origine.»

107 Tн. Herpin, Pronostic et traitement de l'épilepsie. Lancette Française, Gazette des Hôpitaux civils et militaires (Paris) 29 (1856) 502-503, 502.

108 Th. Henpin, Etudes sur le lactate de zinc dans l'épilepsie, Paris 1855 (16 erfolgreich mit Zinklaktat behandelte Fälle); TH. Herpın, Du sélin des marais dans l'épilepsie et quelques autres maladies, Paris 1859 (Erfolge bei der Mehrzahl von 86 Patienten).

109 Th. Herpin, Des accès incomplets d'épilepsie, Paris 1867, S. $110 \mathrm{ff}$. Er wird als Pionier der psychomotorischen Epilepsie auch in modernen Werken anerkannt; vgl. Psychiatrie der Gegenwart (herausgegeben von H.W.GruHLE, R. Jung, W.MAYER-Gross und M. Müller), Band 2, S. 661, Berlin, Göttingen und Heidelberg 1960. Auch sein letztes Werk hat Herpin dem Meister Louis gewidmet, mit dem Zusatz: «Je me suis efforcé dans ce travail de me rendre digne de l'affection et de l'estime dont vous (sc. Louis) voulez bien m'honorer.» 
Die zwei Mitglieder der «Société Médicale d'Observation» aus der übrigen Schweiz, Jules Cossy (1818-1878) aus Lavey und Hermann Lebert (1813-1878) in Zürich, lieferten Beiträge mit guten Beobachtungen, aber ohne weitergehende Anwendung der Zählmethode, zum dritten Band der Mémoires de la Société Médicale d'Observation ${ }^{110}$.

Zusammenfassend läßt sich sagen, daß die Genfer Louis-Schüler nicht die zum Teil spektakulären Ergebnisse ihrer amerikanischen Kollegen erreichten, woran vor allem der Umstand schuld sein dürfte, daß Genf für die Anwendung der von ihnen gelernten Methode einfach zu klein war; die Amerikaner waren diesem Nachteil weniger unterworfen und konnten ferner während heftiger Epidemien ausreichend große Fallzahlen sammeln. Immerhin haben die drei Genfer d'Espine, Maunoir und Bizot der Methode des verehrten Meisters eine Institution geschaffen, wo sie weiterentwickelt und verbreitet werden konnte, nämlich die «Société Médicale d'Observation » mit ihren Mémoires ${ }^{111}$. D'Espine kann (zusammen mit Danvin) zu den frühesten Theoretikern und Verteidigern der Zählmethode gerechnet werden und ist in diesem Sinn ein Vorläufer von Bartlett.

Ferner haben fast alle hier erwähnten Genfer Ärzte noch vor ihrer Abreise aus Paris die numerische Methode praktisch erprobt und in Dissertationen und anderen Arbeiten die damit erzielten Resultate beschrieben. Dank der Verwendung der damals modernsten Methode (die erst durch Histologie, Bakteriologie, experimentell-physiologische Grundlagenforschung sowie Einführung der statistischen Methodenlehre in die Medizin verdrängt wurde) aus dem führenden medizinischen Zentrum der Welt auf Krankheitsbeschreibung, Therapie, Hygiene u.a.m. gelangen ihnen einige beachtliche Werke von hohem Wert - und dies in einer Stadt ohne Universität! Dazu können wir vor allem rechnen Bızots Recherches sur le cour et le système artériel chez l'homme (1837), RiLliets Traité clinique et pratique des maladies des enfants (1843), Herpins Du pronostique et du traitement curatif de l'épilepsie (1852), D’Espines Essai de statistique mortuaire comparée (1858),

110 J. Cossy, Mémoire sur une cause encore peu connue d'engouement interne de l'intestin, Mémoires de la Société Médicale d'Observation 3 (1856) 50-128.

J. Cossy, Recherches sur le délire aigu des épileptiques, ibidem, S. 295-390.

H. Lebert, Mémoire sur les cancroides cutanés, ibidem, S. 180-294. Cossy war Badearzt in Lavey von 1847-1869 sowie Begründer und ärztlicher Leiter des «Hôtel des Salines » in Bex von 1870 bis 1878.

111 Den ersten Band der Mémoires bestreiten denn auch D'Espine, Maunorr und Bizot gemeinsam mit Louis. 
Sestiers De la foudre (1866), Herpins Des accès incomplets d'epilepsie (1867) und Lombards Climatologie médicale (1877-1880). Es sind zweifellos zu einem guten Teil die Arbeiten der hier erwähnten Louis-Schüler, die Genf den kurz vor 1800 erworbenen Ruf als bestes medizinisches Zentrum der Schweiz bis weit über die Mitte des 19. Jahrhunderts hinaus erhalten haben.

Herrn Dr. Marc Cramer, Direktor des «Musée d'Histoire des Sciences» in Genf, danke ich bestens für seine Hilfe bei der Beschaffung des Materials. 